\title{
6 Single Neurons and Primate Behavior
}

\author{
Robert H. Wurtz and Marc A. Sommer
}

Understanding the brain mechanisms mediating cognitive behavior requires combining two experimental steps. First, the brain must actually be engaged in the cognitive behavior under study. Second, the brain activity must be measured during this behavior and the recording sufficiently described to permit replication of the experiment. There are a number of ways of meeting the second prerequisite, many discussed elsewhere in this volume; our chapter will address one with unsurpassed spatial and temporal resolution: the recording of the action potentials of single neurons.

Recording single neurons in the brain is a mature technique that has been used extensively for over a quarter century. In outlining the technique's requirements and comparing them to those of other techniques, we will focus on what has become a cornerstone for the study of brain mechanisms underlying cognitive behavior: single-neuron recording from awake monkeys trained on behavioral tasks. Our description and comments are based on our own experience in studying awake monkeys; we also provide references on specific technical points not addressed in this chapter.

\section{Development}

The ability to study the relation of the brain's neural activity to behavior progressed from the outside in, starting with the discovery that the brain's electrical activity could be recorded outside the skull using techniques that evolved into the field of electroencephalography. From the time of Hans Berger's report of the changes in brain activity during sleep and wakefulness in the 1920s (see Brazier, 1961) until the late 1950s, the electroencephalogram (EEG) was the major window into the electrical activity of the brain. This technique was extended to include changes in brain activity in a range of cognitive states including perception, learning, and memory. A series of symposia in the late fifties and early sixties that summarized many 
of the research directions relating the EEG to cognitive behavior, also noted there were still unresolved problems in using the EEG (see, for example, Wolstenholme, 1958; Jasper and Smirnov, 1960; Fessard, Gerard, and Konorski, 1961). Throughout the era when changes in EEG activity were correlated with behavior, it was uncertain exactly what the EEG was measuring. Was it the summation of action potentials conveyed within a cortical region-or of those between regions? Was it the summation of synaptic potentials-or of dendritic potentials? Moreover, whatever was being measured, it most likely included activity from brain regions substantially larger than those intended, and probably even failed to distinguish between cortical and subcortical activity.

Moving within the skull to study the activity of single neurons solved these problems. The action potential was well understood, and hence there was no ambiguity as to what was being compared to behavior. In addition, because single-neuron activity could only be "seen" within a few hundred microns of its origin, its local origin was assured.

But, in solving one set of problems, single-neuron recording introduced another: how to move the recording electrode near enough to a neuron to record its activity and how to continue this recording during behavior. Jasper, Ricci, and Doane (1958), recognized as the first to record single-neuron activity in an awake, behaving mammal, came up with an ingenious solution-head-mounted microdrives that enabled the investigators to move the electrode while their monkeys performed conditioned response tasks. This was soon followed by other single-neuron research with awake, behaving animals: Hubel (1959, 1960; Hubel et al., 1959) recorded neuronal activity in the visual pathway and auditory cortex of the cat, and Olds (1963) recorded neuronal activity in the rat brain. Figure 6.1 shows an example of one of these early recordings. Further critical advances proceeded from the work of Evarts (Evarts, 1966a, 1968a, 1968b), who pioneered a set of reproducible, highly influential techniques to record from single neurons in the awake monkey.

Equally important, Evarts developed methods for humane, painless restraint; this was critical because, unlike cats or rats, monkeys can simply reach up and dismantle attachments for recording with their fingers. Evarts's detailed description of procedures for recording from single neurons in awake monkeys was the major stimulus for the expansion of recording in awake monkeys that has flourished into the present. Even though almost all of the specific procedures have been extensively modified in laboratories throughout the world, the basic methods were laid out in the three papers cited above, with Evarts's own early experiments 


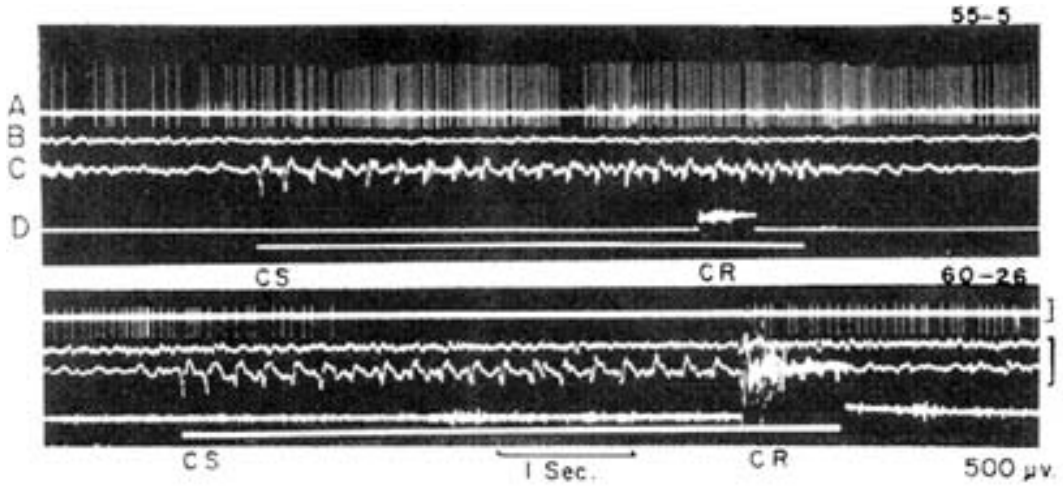

Figure 6.1

Sample of one of the earliest single-neuron recordings from an awake monkey. Records show $(A)$ single-neuron activity, and $(B$ and $C$ ) surface electrocorticogram from motor and visual cortex, respectively, $(D)$ electromyogram of monkey's response, and time of conditioned stimulus (CS) and conditioned response (CR). (From Jasper, Ricci, and Doane 1960)

demonstrating the efficacy of the procedures during sleep, wakefulness, and arm movements (Evarts, 1966a, 1966b). These procedures were promptly extended from motor cortex to the oculomotor neurons (Fuchs and Luschei, 1970; Robinson, 1970; Schiller, 1970).

But recording from a single neuron addresses only half the issue; the other half is comparing neuronal activity to behavior, particularly to more complex cognitive behavior. Although many of the initial single-neuron studies simply compared the neuronal activity with spontaneous behavior, Evarts (1966b) showed that he could elicit the same behavior reliably by training monkeys to do controlled movements, allowing him to repeatedly compare the activity of a single neuron to identical behavior. Given the larger minute-to-minute variability in neuronal activity in the awake versus the anesthetized monkey, this was a critical advance in evaluating the consistency of the correlation between neuronal activity and behavior.

Whereas it is impossible to record neuronal activity related to movement from anesthetized, paralyzed animals, it is both possible and even desirable to record neuronal activity related to sensory responses from such animals, simply because they cannot move. Primarily for this reason, application of single-neuron recording techniques in awake, behaving animals came onto the scene later in sensory than in motor research. Difficulties in using behaving primates were particularly acute for the visual system because, in the awake monkey, the eyes make rapid or saccadic eye 
movements several times per second. Repeatedly directing the visual stimulus to the same place on the retina was therefore not feasible. This problem was solved by using behavioral training techniques that required the monkey to continue fixating at a point on the screen in front of it for several seconds, during which time a visual stimulus could be presented to determine the location of the visual receptive field of the neuron under study (Wurtz, 1969). Again the techniques used to achieve this visual fixation have evolved substantially, especially with improvements in eye movement recording (Robinson, 1963; Judge, Richmond, and Chu, 1980); the use of visual fixation has proven vital to the burgeoning research into cognitive processing in the visual system.

Thus, by the early seventies, the essential techniques required for neuronal recording in awake monkeys were largely worked out and the opportunities for investigating the mechanisms of cognitive processing in the awake monkey had become established. Subsequently, several improved techniques have converted recording in awake monkeys from a harrowing to a standard, albeit challenging, procedure. At the behavioral level, the change has been even more striking: the application of behavioral techniques has transformed the nature of the questions that can be asked in monkey experiments and has opened the door to the analytical study of cognitive questions at the single-neuron level. The old worry many investigators had about whether anything could be learned studying single neurons in the midst of the deluge of neuronal activity in awake, behaving animals has been dispelled; the new worry is whether the technique can address larger issues of cognitive brain function.

\section{Principles}

Because, as we noted, single-neuron recording is a mature technique, there are now multiple solutions to many of the technical problems, detailed explanations in the literature, and commercial sources for much of the hardware required. Here we concentrate on the salient principles of the technique, providing references to several especially useful sources of further information for each step.

The most fundamental principle of the method is that the single neurons are recorded extracellularly, with the flow of current generated by the active neurons being detected through the volume of the fluid within the brain. As a consequence, the farther the active neuron is from the electrode the smaller the signal, with the amplitude of the signal declining as the cube of the distance between neuron and electrode. Furthermore, the 
extracellular fluid acts like a low-pass filter so that the high-frequency band containing action potential signals is reduced the most and the lowerfrequency bands are reduced the least. Thus, to isolate the action potentials of a neuron from its neighbors, the electrode tip has to be close to the neuron. The good news is that when the activity of the neuron is isolated from the background activity, we can be certain it relates to the local area under study. The bad news is that larger neurons-which may or may not be more important functionally-tend to be recorded preferentially, leading to inherent sampling biases (a fact rarely discussed by investigators). Although lower frequencies can also be recorded, because they travel over considerably longer distances, they cannot be so narrowly localized.

\section{Basic Methods}

As the interface between the brain and the registration of the brain activity, the microelectrode has understandably been a much-investigated topic since the dawn of the single-neuron recording technique. Because of their sturdy structure, metal microelectrodes have been preferred; various metals and alloys have been used, including steel (Green, 1958), tungsten (Hubel, 1957), and platinum-iridium (Wolbarsht, MacNichol, and Wagner, 1960). Although initially made within the laboratory, microelectrodes are now produced commercially and distributed by a number of vendors (see http:// www.fh-co.com/ and http://www.thomasrecording.com/pdf/Elektroden.pdf). Basic variables in the microelectrode are the size and shape of the tip exposed to the brain, although the relative importance of these variables remains a matter of discussion. For practical reasons, the impedance of the microelectrode is usually taken as a measure of its quality. The range for metal microelectrodes falls between 100 kilohms and several megohms (at $1,000 \mathrm{~Hz}$ ): the higher the impedance, the better the isolation, although the fewer neurons isolated, the greater the difficulty in holding the neuron. The microelectrodes are introduced into the brain using microdrives to advance or retract them to the desired depth; these drives are equipped with a coarse control in the millimeter range to move to the approximate depth, and a fine control in the micron range to move to the precise depth. Because of the compliance of the brain to the push of the microelectrode, the smallest step we usually make is five microns. The microelectrode must also be advanced or retracted remotely, so that moving it does not disrupt the monkey's behavior, and positioned not only upward and downward but also in the $x-y$ plane over the brain, so that one actually targets the brain structure of interest. The microdrive has evolved over the years, and a 
variety of hydraulic and electrical motor devices are now available commercially (see http://www.fh-co.com/; http://www.alphaomega-eng.com/; for construction from drawings, see http://www.lsrweb.net). Although screw drives are occasionally used because of their simplicity and small size, most cannot be controlled remotely (see, for example, Nichols et al., 1998)

The microdrive is typically attached to the monkey only during a recording session, using an implanted base that is screwed or cemented to the skull, or both, in a previous surgery done under general anesthesia. During that initial surgery, the skull inside the base is removed so that the dura is exposed and electrode penetrations can be made into the brain through the dura. We use a circular base into which we insert a grid with holes drilled at one millimeter intervals (see Crist et al., 1988); through these holes, we pass a stainless steel guide tube that either rests close to the surface of the dura or penetrates the dura to allow access to deeper regions of the brain. The grid is at least $0.5 \mathrm{~cm}$ thick to ensure that microelectrodes inserted into different holes will follow parallel paths into the brain. We leave the dura intact because it reduces the risk of infection when the recording extends for many months. Alternatively, the dura can be removed and an artificial dura installed (see Arieli, Grinvald, and Slovin, 2002), or only small holes can be drilled in the skull so that a microelectrode always passes through newly exposed dura.

The hardware used to attach the microdrive to the skull and to attach brackets to restrain the head varies widely between laboratories. The hardware developed in our laboratory is typically made from plastic for magnetic resonance imaging (MRI) compatibility. Figure 6.2 shows examples of the hardware we currently use (for a description, see our Web site, http://www.lsr-web.net) and is commercially available (see http://www.cristinstrument.com).

Because the neuron is being recorded at a distance, identification of a single neuron as opposed to several is critical. There are basically two steps in this identification. The first is to observe the change in amplitude and shape of the recorded action potential as the electrode is advanced or retracted. Since the neurons are at different distances from the electrode, movement of the electrode shortens the distance from some neurons and the spike from those neurons becomes larger. A variant of this approach is the use of a tetrode, four separate recording electrodes typically cemented together and applied to four closely spaced recording sites (see Gray et al., 1995). The second step is to discriminate individual neurons based on the amplitude, shape, or both, of their action potentials. The simplest discriminators select individual neurons on the basis of spike amplitude; the 


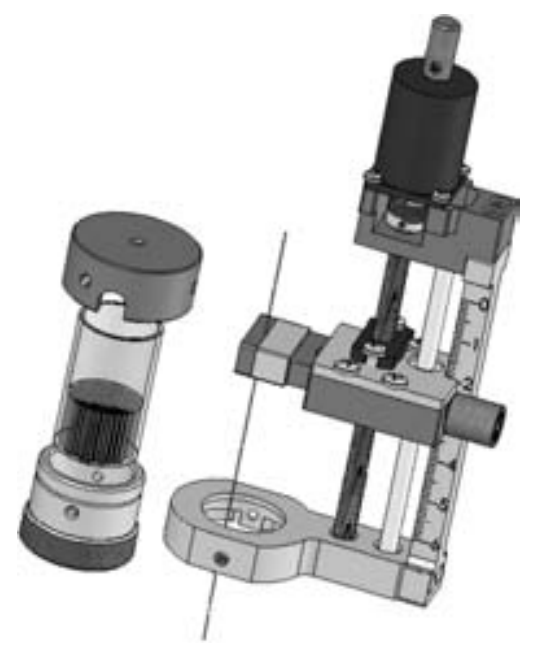

Figure 6.2

Example of single-neuron recording apparatus. On the left, in an exploded view, is an implanted base, a grid that fits into the base, and a cap that covers the assembly when the microdrive is not attached. On the right is a microelectrode held by a microdrive with a stepper motor that advances the microelectrode. (Illustration courtesy of Altah M. Nichols and Thomas W. Ruffner)

addition of multiple thresholds for different phases of the spike and of a temporal window for width of the spike waveform makes the discrimination more powerful. Other methods, such as relying on principal component analysis for the identification, permit discrimination based on criteria other than spike amplitude and width, although such analysis usually requires that discrimination be made after a sample of spikes has been obtained, that is, after the behavior has been completed. Discrimination by spike amplitude and width frequently also benefits from post hoc analysis after preliminary online analysis.

Location of the region of the brain to be studied is now greatly aided by making an MRI of the monkey's brain before the experiment begins. In our case, we usually place the recording cylinder base on the skull, install the grid, and insert several tungsten microelectrodes (for a $1.5 \mathrm{~T}$ magnet and without the stainless steel guide tubes) into the brain to about a centimeter above where we plan to record. If steel electrodes are used, small marks made by passing current can be seen on the MRI (see Fung, Burstein, and Born, 1998). This allows us to shift our recordings on the grid so that the penetrations are centered on the desired target and to more accurately estimate depth for subcortical targets. 
After the experiment, the site of the neurons in the brain can be histologically located by a mark placed at the recording site, usually by passing a 10-20 $\mu \mathrm{A}$ current through the microelectrode depending on the expected survival time of the monkey, the number of marks in the area, and the required precision of the localization. This localization of the recording site is a key requirement of single-neuron recording: an experiment can be replicated only if one accurately determines where the original neurons were. Recovery of the marks is typically accomplished by inspecting alternating cell and fiber stained histological sections no thicker than 50 microns. We frequently code penetrations with a pattern of marks higher up in the penetration as we withdraw the electrode.

We can hardly conclude our consideration of basic methods without emphasizing that the power of single-neuron recording has been immeasurably enhanced by two technical advances. First and foremost, the advent of cheap but powerful computers has enabled the development of sophisticated and easily altered programs for controlling behavioral paradigms and stimulus presentations, recording behavioral and neuronal responses, displaying experimental data online, and analyzing experimental results offline. Second, the availability of structural MRI to verify the recording site, at least approximately, during the experiment has allowed us to explore both new brain areas and multiple areas at the same time, a feat that was exceedingly difficult in the pre-MRI era.

\section{A Trilogy of Techniques}

Our description of the basic methods cannot help but leave the impression that the end goal is simply to compare neuronal activity to behavior. But such a goal yields only a correlation of neuronal activity at one point in the brain with a specific cognitive behavior. Although a good start, it is still just a correlation and, as such, would not tell us the degree to which the neurons are part of the brain mechanisms producing behavior. A strength of the single-neuron recording technique is that it can be paired with two other techniques that move it beyond the realm of correlation: stimulation and reversible inactivation of the neurons at the recording site. We refer to the set of techniques-neuronal recording, stimulation, and inactivationas the "trilogy of techniques," a trilogy that moves single-neuron neurophysiology from the observation of correlations into the study of causation.

Our general approach is to formulate hypotheses about the relation of the neuronal activity to behavior based on single-neuron recordings and 
then test these hypotheses by altering this neuronal activity by activating or inactivating the neurons.

Neurons can be activated by using an excitatory transmitter or by blocking the continuing action of an endogenous inhibitory transmitter; we have used bicuculline to block the GABA receptors. Alternatively, activation can be accomplished by electrical stimulation of the neurons, by passing an electrical current through the same microelectrode used to record the activity, a method used extensively in cognitively related experiments (Tehovnik, 1996; Cohen and Newsome, 2004). The advantage of electrical stimulation is that, whereas transmitter activation and inactivation alter neuronal activity over a period of time, electrical stimulation can provide activation trial by trial. For inactivation, the optimal method is to suppress the neuronal activity by applying an inhibitory transmitter, and we have relied on the inhibitory transmitter GABA, using either GABA itself or its agonist muscimol. The advantage of the GABA agonist over an anesthetic is that it inactivates only the local neurons but not the fibers of passage (which have no receptors), whereas an anesthetic acts on both (see Lomber, 1999). A promising future direction is the activation or inactivation of a specific subset of neurons using molecular biological techniques (see Tan et al., 2003).

Both activation and inactivation have substantial limits on their interpretation. Activation using electrical stimulation raises questions as to what neurons are altered and at what distance, the extent to which remote neuronal activity is invoked by the stimulation of axons, and the artificial conjunction of activity in neurons that are never normally active together. Inactivation using transmitter agonists, on the other hand, raises questions as to how far the agonist spreads and what neurons are affected by the spread.

Although, taken individually, each of the trilogy of techniques has limitations, used in a coordinated manner, the trilogy is powerful because each technique overcomes at least to some degree the other techniques' limitations. Judicious use allows experimenters to push beyond correlation to causation, maximizing the opportunity available to them.

\section{Innovation}

Everyone doing single-neuron recording would happily provide a wish list for innovations that would expand the technique. Here we consider two innovations that address two major problems with single-neuron recording: (1) multielectrode recording, more often referred to as "multichannel 
recording," to overcome the limitation in area of brain region studied; and (2) anti- and orthodromic stimulation to overcome the difficulty of identifying the connections of neurons studied.

Multichannel recording addresses a central feature of single-neuron recording that may also be considered its central deficiency: recordings are made from only neuron at a time. By introducing multiple electrodes into the brain, more than one neuron can be recorded within a structure or from several different structures. The advantages of multichannel recording are in sampling a larger number of neurons within a population, differentiating the extent to which these neurons are related to a given behavior trial by trial, examining the cross-correlation between neurons, and recording a larger number of neurons on any given set of trials. One method of obtaining these multiple recordings is to insert chronic wire electrodes into the brain (see deCharms, Blake, and Merzenich, 1999; Nicolelis et al., 2003), and this has been used to compare activity between areas along the somatosensory pathway (see, for example, Faggin et al., 1997) and within structures such as motor cortex or the superior colliculus (see Maynard et al., 1999; Hanes et al., 2005). This method has the advantage of allowing experimenters to insert many bundles of electrode wires and to record at many sites. Multichannel systems that record up to 128 channels are available commercially (see http://www.fh-co.com or http://www.alphaomegaeng.com). A disadvantage is the inability to move the electrode, which means that the neurons found are the ones that must be studied, and also that one of the two ways in which unit isolation is verified is lost. A method that preserves the ability to move the electrodes individually is to attach each to a separate micromanipulator. This method has the advantage of verifying the isolation of single neurons and selecting those from the population of interest, but the disadvantage of recording from comparatively fewer neurons. Both multichannel methods introduce the difficulty of characterizing each neuron using tests that could zero in on their individual traits, a difficulty that often results in such characterizations not being made or in a battery of tests being applied to the whole sample en masse. For example, running a fixed pattern of targets for eye movements enables experimenters to identify the visual receptive fields or movement fields of neurons, but how well the field of each neuron is determined depends on how closely spaced the targets are (see Hanes et al., 2005). In general, multichannel recordings are difficult to apply in areas where neurons related to disparate parts of visual or motor space are clustered near each other (such as prefrontal cortex); they are better suited to regions that contain highly pre- 
dictable maps. The underlying risk in multichannel recordings is that one sacrifices quality (careful characterization of each neuron) for the sake of quantity (a spectacular increase in sample size).

Although experimenters need of course to know where a neuron is located in the brain, they also need to understand its connections. Identification of at least some of these connections can be made through the use of antidromic and orthodromic stimulation, a standard practice in experiments on anesthetized animals but one rarely adopted for experiments on awake, behaving animals. Interestingly enough, however, in some of the first single-neuron recordings made from awake, behaving monkeys, neurons in the skeletomotor and oculomotor frontal cortex were identified as descending output neurons by antidromically stimulating them from lower areas (Evarts, 1966b; Bizzi, 1968). We have recently combined antidromic with orthodromic stimulation to identify relay neurons in the pathway from superior colliculus in the brain stem through the mediodorsal nucleus of the thalamus to the frontal eye field in frontal cortex. Antidromic stimulation from frontal cortex showed that a mediodorsal neuron projected to the frontal cortex, while orthodromic stimulation from the superior colliculus showed that the same neuron received input from the superior colliculus (figure 6.3; Sommer and Wurtz, 2004a, 2004b). Antidromic stimulation is straightforward and simple and relies on the regular, short latency of the antidromic response in the recorded neuron. In contrast, because the orthodromic response in the recorded neuron is subject to multiple interpretations-synaptic activation can take numerous routesorthodromic stimulation requires added precautions. (These issues are considered in detail in the excellent review by Lemon, 1984.)

A second advantage of antidromic stimulation is that it can correct for sampling biases. Recording as the microelectrode passes through the brain has been compared to shooting fish in a barrel: the larger the fish, the more likely it is to be hit (see Towe and Harding, 1970). With antidromic stimulation, the conduction speed of a neuron can be calculated. Faster speeds imply larger axons and neuronal cell bodies, and therefore estimates of the relative cell sizes can be made. Knowing this distribution of cell sizes allows one to determine the actual distribution of neuronal classes in an area (in terms of the signals they carry) and not just their observed, biased distribution (Humphrey and Corrie, 1978). For example, the seeming prevalence of neurons with activity related to eye movements in the frontal eye field turns out to be, in part, illusory; corrections for sampling bias indicate that such neurons are larger than their neighbors (Sommer and Wurtz, 2000). 


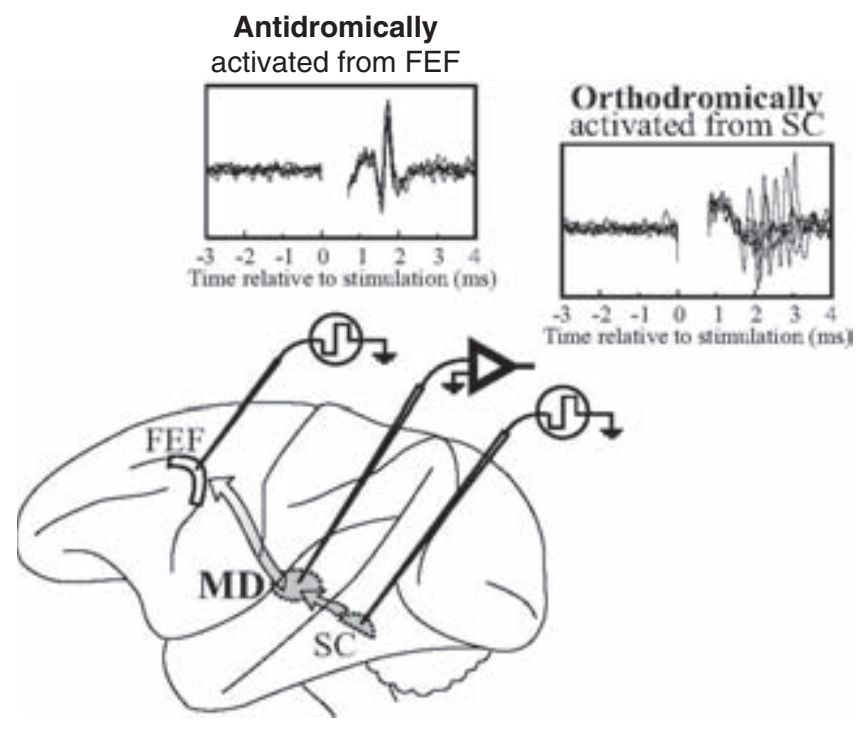

Figure 6.3

Example of antidromic and orthodromic stimulation as used to identify connection of neurons. Stimulating electrodes were implanted with their tips in the frontal eye field (FEF) and the superior colliculus (SC). A single neuron was recorded in the mediodorsal thalamus (MD). At top left, several of the MD neuron's action potentials are aligned to the start of stimulation (at time 0 ) in the FEF. The highly repeatable activation at invariant latency is indicative of antidromic activation (showing that the neuron projects to the frontal eye field). At top right, action potentials of the same mediodorsal thalamus neuron are aligned to the start of superior collicus stimulation. The jittery activation latency is indicative of orthodromic activation (showing that the neuron receives input from the superior collicus). (After Sommer and Wurtz, 2004a)

\section{Limitations}

The principal limitation of single-neuron recording in cognitive studies is glaringly obvious: studying one neuron at a time in a brain mass of billions of neurons. No behavior we are aware of in vertebrates results from the activity of a single neuron or of small, localized groups of neurons. Even machinelike functions such as the stabilization of the visual scene by ocular following, which has one of the shortest latencies in the primate visual system $(70 \mathrm{msec}$ at most), probably arise from the activity of a population of cortical (Takemura et al., 2001) and subcortical neurons. Few would doubt that more cognitive functions such as visual perception and the planning of action must result from the activity of far larger populations of neurons over a series of steps in the brain. 
A possible solution to this problem is the growing use of functional magnetic resonance imaging to assess the activity of brain regions during cognitive behavior. The problem of activity related to only a few neurons is solved: the fMRI indicates phases of the hemodynamic response secondary to neuronal activity, and thus of necessity covers a large population of neurons. The noninvasive nature of fMRI also permits researchers to study these populations of neurons in the human brain during demanding cognitive tasks. The disadvantage is that the fMRI does not measure electrical activity directly, and how the hemodynamic response correlates with various phases of activity (e.g., synaptic potentials vs. action potentials) remains controversial. Moreover, this hemodynamic response introduces a temporal filter, currently on the order of one second at best, a time that far exceeds that required for many cognitive functions. For example, a subject can look at a scene, select what aspect is important, make an eye movement to it, and repeat this two to three times within the time needed to obtain an fMRI response. For examination of the mechanisms in the brain that underlie cognition, fMRI cannot compete with single-neuron recording. The sequential application of fMRI and single-neuron recording in monkeys, however, holds great promise. Functional MRI could identify regions of the brain that are involved in cognitive processes without any indication of the underlying mechanism, while follow-up single-neuron recordings could decipher the code for those mechanisms.

At the opposite end of the continuum, a second limitation of singleneuron recording is that microcircuitry and local connections (e.g., along the dendrites) cannot be determined with this in vivo, extracellular technique. The synaptic activity of a neuron and its degree of membrane depolarization are simply inaccessible to the extracellular microelectrode. With slice and cell culture preparations, these limitations are overcome. For example, in studying the superior colliculus in rodent brain slices, Hall and Isa and their collaborators (see, for example, Hall and Lee, 1997; Lee et al., 1997; Isa, Endo, and Saito, 1998; Isa and Saito, 2001) have measured the distance over which inhibition can be obtained and the extent to which superficial layers functionally activate the intermediate layers, factors that extracellular recording had not determined over the preceding 30 years. Hall and Lee (1997) have also been able to photoactivate specific identified neurons, which is impossible to do in the behaving monkey. On the other hand, creating a slice cuts the inputs and outputs to the colliculus from the rest of the brain and decouples the activity from its relation to behavior. Again, combining information from both techniques sharpens the insights gained from each. 
The point is that single-neuron recording, though in itself a limited technique, can be a powerful investigative tool when used in conjunction with other, complementary techniques.

\section{Integration}

If we want to know about the brain mechanisms that underlie cognitive behavior, we need to study the smallest individual elements of brain circuits: single neurons. Knowing the detailed characteristics of neuronal activity will require recording from individual neurons both separately and together with others, and the better the connections of these neurons can be determined, the stronger the conclusions that can be drawn. These are all correlations, however, and to move beyond the correlations, it is essential to combine single-neuron recording with stimulation and inactivation of the neurons. The understanding derived from this "trilogy of techniques" in turn can be strengthened by fMRI measurements, which reflect large populations of neurons, and by in vitro approaches, which provide insight into the microcircuitry underlying neuronal activity. Although we do not know what technical breakthroughs lie beyond our horizon, we do know that advances in understanding the brain have always relied on a combination of techniques, and single-neuron recording is no exception.

\section{References}

Arieli, A., Grinvald, A., and Slovin, H. (2002). Dural substitute for long-term imaging of cortical activity in behaving monkeys and its clinical implications. Journal of Neuroscience Methods, 114, 119-133.

Bizzi, E. (1968). Discharge of frontal eye field neurons during saccadic and following eye movements in unanesthetized monkeys. Experimental Brain Research, 6, 69-80.

Brazier, M. A. B. (1961). A history of the electrical activity of the brain: The first half-century. London: Pittman.

Cohen, M. R., and Newsome, W. T. (2004). What electrical microstimulation has revealed about the neural basis of cognition. Current Opinion in Neurobiology, 14, 169-177.

Crist, C. F., Yamasaki, D. S. G., Komatsu, H., and Wurtz, R. H. (1988). A grid system and a microsyringe for single cell recording. Journal of Neuroscience Methods, 26, 117-122.

deCharms, R. C., Blake, D. T., and Merzenich, M. M. (1999). A multielectrode implant device for the cerebral cortex. Journal of Neuroscience Methods, 93, 27-35. 
Evarts, E. V. (1966a). Methods for recording activity of individual neurons in moving animals. In R. F. Rushmer (Ed.), Methods in medical research, 241-250. Chicago: Year Book.

Evarts, E. V. (1966b). Pyramidal tract activity associated with a conditioned hand movement in the monkey. Journal of Neurophysiology, 29, 1011-1027.

Evarts, E. V. (1968a). A technique for recording activity of subcortical neurons in moving animals. Electroencephalography and Clinical Neurophysiology, 24, 83-86.

Evarts, E. V. (1968b). Relation of pyramidal tract activity to force exerted during voluntary movement. Journal of Neurophysiology, 31, 14-27.

Faggin, B. M., Nguyen, K. T., and Nicolelis, M. A. (1997). Immediate and simultaneous sensory reorganization at cortical and subcortical levels of the somatosensory system. Proceedings of the National Academy of Sciences, USA, 94, 9428-9433.

Fessard, A., Gerard, R. W., and Konorski, J. (1961). Brain mechanisms and learning. Springfield, IL: Charles C. Thomas.

Fuchs, A. F., and Luschei, E. S. (1970). Firing patterns of abducens neurons of alert monkeys in relationship to horizontal eye movement. Journal of Neurophysiology, 33, 382-392.

Fung, S. H., Burstein, D., and Born, R. T. (1998). In vivo microelectrode track reconstruction using magnetic resonance imaging. Journal of Neuroscience Methods, 80, 215-224.

Gray, C. M., Maldonado, P. E., Wilson, M., and McNaughton, B. (1995). Tetrodes markedly improve the reliability and yield of multiple single-unit isolation from multi-unit recordings in cat striate cortex. Journal of Neuroscience Methods, 63, 43-54.

Green, J. D. (1958). A simple microelectrode for recording from the central nervous system. Nature, 182, 962.

Hall, W. C., and Lee, P. (1997). Interlaminar connections of the superior colliculus in the tree shrew. 3. The optic layer. Visual Neuroscience, 14, 647-661.

Hanes, D. P., Smith, M. K., Optican, L. M., and Wurtz, R. H. (2005). Recovery of saccadic dysmetria following localized lesions in monkey superior colliculus. Experimental Brain Research, 160(3), 312-325.

Hubel, D. H. (1957). Tungsten microelectrode for recording from single units. Science, 125, 549-550.

Hubel, D. H. (1959). Single unit activity in striate cortex of unrestrained cats. Journal of Physiology, 147, 226-238.

Hubel, D. H. (1960). Single unit activity in lateral geniculate body and optic tract of unrestrained cats. Journal of Physiology, 150, 91-104.

Hubel, D. H., Henson, C. O., Rupert, A., and Galambos, R. (1959). “Attention” units in the auditory cortex. Science, 129, 1279-1280. 
Humphrey, D. R., and Corrie, W. S. (1978). Properties of a pyramidal tract neuron system within a functionally defined subregion of primate motor cortex. Journal of Neurophysiology, 41, 216-243.

Isa, T., Endo, T., and Saito, Y. (1998). The visuo-motor pathway in the local circuit of the rat superior colliculus. Journal of Neuroscience, 18, 8496-8504.

Isa, T., and Saito, Y. (2001). The direct visuo-motor pathway in mammalian superior colliculus: Novel perspective on the interlaminar connection. Neuroscience Research, 41, 107-113.

Jasper, H., Ricci, G. F., and Doane, B. (1958). Patterns of cortical neuronal discharge during conditioned responses in monkeys. In G. E. W. Wolstenholme and Cecilia M. O'Connor (Eds.), Neurological basis of behaviour. London: Churchill.

Jasper, H., Ricci, G., and Doane, B. (1958). Microelectrode analysis of cortical cell discharge during avoidance conditioning in the monkey. In H. H. Jasper and G. D. Smirnov (Eds.), The Moscow colloquium on encephalography of higher nervous activity. Electroencephalography and Clinical Neurophysiology, suppl. 13.

Jasper, H. H., and Smirnov, G. D. (Eds.) (1960). The Moscow colloquium on encephalography of higher nervous activity. Electroencephalography and Clinical Neurophysiology, suppl. 13.

Judge, S. J., Richmond, B. J., and Chu, F. C. (1980). Implantation of magnetic search coils for measurement of eye position: An improved method. Vision Research, 20, 535-538.

Lee, P. H., Helms, M. C., Augustine, G. J., and Hall, W. C. (1997). Role of intrinsic synaptic circuitry in collicular sensorimotor integration. Proceedings of the National Academy of Sciences, USA, 94, 13299-13304.

Lemon, R. (1984). Methods for neuronal recording in conscious animals. IBRO Handbook Series: Methods in the Neurosciences, vol. 4. New York: Wiley.

Lomber, S. G. (1999). The advantages and limitations of permanent or reversible deactivation techniques in the assessment of neural function. Journal of Neuroscience Methods, 86, 109-117.

Maynard, E. M., Hatsopoulos, N. G., Ojakangas, C. L., Acuna, B. D., Sanes, J. N., Normann, R. A., and Donoghue, J. P. (1999). Neuronal interactions improve cortical population coding of movement direction. Journal of Neuroscience, 19, 8083-8093.

Nichols, A. M., Ruffner, T. W., Sommer, M. A., and Wurtz, R. H. (1998). A screw microdrive for adjustable chronic unit recording in monkeys. Journal of Neuroscience Methods, 81, 185-188.

Nicolelis, M. A., Dimitrov, D., Carmena, J. M., Crist, R., Lehew, G., Kralik, J. D., and Wise, S. P. (2003). Chronic, multisite, multielectrode recordings in macaque monkeys. Proceedings of the National Academy of Sciences, USA, 100, 11041-11046.

Olds, J. (1963). Mechanisms of instrumental conditioning. Electroencephalography and Clinical Neurophysiology, suppl. 24, 219-225. 
Robinson, D. A. (1963). A method of measuring eye movement using a scleral search coil in a magnetic field. IEEE Transactions on Biomedical Engineering, 10, 137-145.

Robinson, D. A. (1970). Oculomotor unit behavior in the monkey. Journal of Neurophysiology, 33, 393-404.

Schiller, P. H. (1970). The discharge characteristics of single units in the oculomotor and abducens nuclei of the unanesthetized monkey. Experimental Brain Research, 10, 347-362.

Sommer, M. A., and Wurtz, R. H. (2000). Composition and topographic organization of signals sent from the frontal eye field to the superior colliculus. Journal of Neurophysiology, 83, 1979-2001.

Sommer, M. A., and Wurtz, R. H. (2004a). What the brain stem tells the frontal cortex. 1. Oculomotor signals sent from superior colliculus to frontal eye field via mediodorsal thalamus. Journal of Neurophysiology, 91, 1381-1402.

Sommer, M. A., and Wurtz, R. H. (2004b). What the brain stem tells the frontal cortex. 2. Role of the SC-MD-FEF pathway in corollary discharge. Journal of Neurophysiology, 91, 1403-1423.

Takemura, A., Inoue, Y., Kawano, K., Quaia, C., and Miles, F. A. (2001). Singleunit activity in cortical area most associated with disparity-vergence eye movements: Evidence for population coding. Journal of Neurophysiology, 85, 2245-2266.

Tan, E. M., Horwitz, G. D., Albright, T. D., and Callaway, E. M. (2003). A genetic method for selective and quick reversible silencing of mammalian neurons in vivo. Society of Neuroscience Abstracts, 29, 125.122.

Tehovnik, E. J. (1996). Electrical stimulation of neural tissue to evoke behavioral responses. Journal of Neuroscience Methods, 65, 1-17.

Towe, A. L., and Harding, G. W. (1970). Extracellular microelectrode sampling bias. Experimental Neurology, 29, 366-381.

Wolbarsht, M. L., MacNichol, E. F. J., and Wagner, H. G. (1960). Glass insulated platinum microelectrode. Science, 132, 1309-1310.

Wolstenholme, G. (1958). The neurological basis of behavior. Boston: Little, Brown.

Wurtz, R. H. (1969). Visual receptive fields of striate cortex neurons in awake monkeys. Journal of Neurophysiology, 32, 727-742. 